\title{
Correction: Ali, M., et al. Study on the Development of Neutrosophic Triplet Ring and Neutrosophic Triplet Field. Mathematics 2018, 6, 46
}

\author{
Yılmaz Çeven ${ }^{1}(\mathbb{D})$ and Florentin Smarandache ${ }^{2, *(1)}$ \\ 1 Department of Mathematics, Süleyman Demirel University, 32260 Isparta, Turkey; yilmazceven@sdu.edu.tr \\ 2 Department of Mathematics, University of New Mexico, 705 Gurley Avenue, Gallup, NM 87301, USA \\ * Correspondence: fsmarandache@gmail.com
}

Received: 5 May 2019; Accepted: 19 June 2019; Published: 24 June 2019

We have found the following errors in the article which was recently published in Mathematics [1]:

1. In Example 1, 3 gives rise to the neutrosophic triplet $(3,3,3)$. However, 3 has two neutrals: neut $(3)=\{3,5\}$, but 3 does not give rise to a neutrosophic triplet for neut $(3)=5$, since anti(3) does not exist in $\mathbb{Z}_{6}$ with respect to neut $(3)=5$.

2. In Example 2, $\mathbb{Z}_{10}$ is not a neutrosophic triplet group. 7 is the classical unitary element of the set $\mathbb{Z}_{10}$. Therefore $\mathbb{Z}_{10}$ is a neutrosophic extended triplet group.

3. In classical ring theory, for any ring $(R,+,),$.0 is the additive identity element. However, in a neutrosophic triplet ring $(N, *, \#), 0$ is an ordinary element and the element 0 is not used in definition. Also $\mathrm{N}$ may not have such an element. So, in Definition 8 and subsequent parts of the paper, when using the element 0 , the element 0 should be defined.

4. In classical ring theory, for any ring $(R,+,),. n \cdot a$ is defined by $a+\ldots+a$ and $a^{n}$ is defined by $a \ldots a$ (n times). In neutrosophic triplet ring (NTR), we do not know the definition of $a^{n}$. So before Definition 11, the element $a^{n}$ should be defined.

5. For the proof of Theorem 3, Theorem 1 was used. So Theorem 3 must satisfy the hypothesis of Theorem 1. Also according to definition of $a^{n}$, Theorem 3 should be rewritten.

6. Proposition 1 and its proof are not true. The sentences "if a is not a zero divisor, so a is cancellable" and "if a is cancellable, a is not a zero divisor" are not true. These statements cannot be obtained from the given definitions and theorems.

7. The set $P(X)$ in Example 3 is not neutrosophic triplet field. $P(X)$ has identity elements $X$ and $\varnothing$ for the operations $\cup$ and $\cap$, respectively. Therefore $P(X)$ is a neutrosophic extended triplet group.

8 . The counterexamples given for Theorem 5 do not satisfy the distributive law since $1 \#(1 * 2) \neq$ $(1 \# 1) *(1 \# 2)$.

9. In the proof of Theorem 6 , the set $\mathrm{N}$ is not NTF since $5 \#(5 * 5) \neq(5 \# 5) *(5 \# 5)$.

10. The proof of Theorem 7(2) is not true. If $c \in U$, then $f^{-1}(c)$ is a set. If $\mathrm{f}$ is not a function, $f^{-1}(c)$ can be equal to an empty set. Then $f^{-1}(c) * f^{-1}(d)$ is not in $f^{-1}(U)$. We can prove it by the following:

Let $a, b \in f^{-1}(U)$. Then $f(a), f(b) \in U$ and $f(a) \oplus f(b)=f(a * b) \in U$. Hence we get $a * b \in f^{-1}(U)$. The proof of $a \# b \in f^{-1}(U)$ is similar. Also, since $f(a) \in U$ and neut $*(f(a))=f($ neut $*(a)) \in U$, we have neut * $(a) \in f^{-1}(U)$. The proof of neut $t^{\#}(a) \in f^{-1}(U)$ is similar.

11. The proof of Theorem 7(3) is not true. If $i \in I$ and $r \in N T R_{2}$, then $f^{-1}(i)$ and $f^{-1}(r)$ is a set. If $f$ is not a function, $f^{-1}(i)$ and $f^{-1}(r)$ can be equal to an empty set. Then $f^{-1}(i) * f^{-1}(r)$ is not in $f^{-1}(I)$. We can prove it by the following:

Let $a \in f^{-1}(U)$ and $r \in N T R_{1}$. Then $f(a) \in I$ and $f(r) \in N T R_{2}$ and $f(a) \oplus f(r)=f(a * r) \in I$. Hence we get $a * r \in f^{-1}(I)$. The remaining part of the proof is similar.

12. The proof of Theorem 7(4) should be proven as the following: 
Let $j \in f(J)$ and $r \in N T R_{2}$. Since $f$ is onto, then $\exists h \in J$ exists such that $f(h)=j$ and $\exists s \in N T R_{1}$ such that $f(s)=r$. Then $h * s \in J$ and we get $f(h * s)=f(h) \oplus f(s)=j \oplus r \in f(J)$.

\section{Reference}

1. Ali, M.; Smarandache, F.; Khan, M. Study on the Development of Neutrosophic Triplet Ring and Neutrosophic Triplet Field. Mathematics 2018, 6, 46. [CrossRef] 